\title{
Idade, maturação sexual, variáveis antropométricas e composição corporal: influências na flexibilidade
}

\author{
Influence of age, sexual maturation, anthropometric variables \\ and body composition on flexibility
}

1 Especialista em Treinamento Desportivo e Personalizado. Cascavel, PR. Brasil.

2 Faculdade Assis Gurgacz. Cascavel, PR. Brasil

3 Universidade Estadual de Londrina, PR. Brasil

4 Mestre Educação Física. Linha de pesquisa Cineantropometria. Cascavel, PR. Brasil.

Recebido em 26/09/09 Revisado em 04/11/09 Aprovado em 17/12/09
Resumo - Considerando a flexibilidade uma importante variável da aptidão física relacionada à saúde para todas as idades, torna-se necessário avaliá-la em crianças e adolescentes, pois a capacidade de adquirir e manter índices de flexibilidade é maior nesta faixa etária. $O$ estudo teve como objetivo comparar a massa corporal, estatura, índice de massa corporal e flexibilidade com a idade cronológica e a maturação sexual e verificar a influência das variáveis, avaliadas neste estudo, na flexibilidade de escolares da rede pública e particular de ensino. Este estudo caracteriza-se por ser de natureza descritivo analítico transversal. A amostra foi composta por 2604 meninas de oito a 17 anos, sendo avaliada a massa corporal, estatura, IMC, maturação sexual e flexibilidade. Foi empregada uma análise descritiva dos dados. Os testes inferências utilizados foram: análise de variância (Anova Two-way) seguida do teste de Post Hoc de Tukey os coeficientes de correlação simples de Pearson e Spearman e a análise de regressão linear múltipla "stepwise". Todos os procedimentos estatísticos foram realizados no programa SPSS ${ }^{\circledR} 13.0$, com nível de significância de $p<0,05$. As diferenças estatisticamente significativas ocorreram com o avanço da idade e dos estágios maturacionais para as variáveis de massa corporal, estatura e IMC, quando comparados a partir de um ano mais velho. Conclui-se que com o avanço da idade as variáveis de massa corporal e estatura sofrem aumento crescente, principalmente, nas idades compreendidas entre oito a 13 anos, e a flexibilidade manteve-se estável por toda a infância e adolescência.

Palavras-chave: Escolares; Flexibilidade; Maturação; Composição corporal.

Abstract - Since flexibility is an important component of health-related physical fitness at all ages, this parameter should be evaluated in children and adolescents because the ability to acquire and maintain levels of flexibility is greater in this age group. Thus, the objective of this study was to evaluate body weight, height, BMI and flexibility according to chronological age and sexual maturation and to determine the influence of these variables on flexibility in students from public and private schools. A cross-sectional descriptive and analytical study was conducted. The sample consisted of 2604 girls aged 8 to 17 years. Body weight, height, BMI, sexual maturation, and flexibility were evaluated. The data were analyzed descriptively using the following inferential tests: two-way analysis of variance (ANOVA) followed by the Tukey post-hoc test, Pearson's and Spearman's simple correlation coefficients, and stepwise multiple regression analysis. The SPSS 13.0 program was used for all statistical analyses, with a level of significance of $p<0.05$. Significant differences with increasing age and maturation stage were observed for the variables body weight, height and BMI when compared to the subsequent year. In conclusion, body weight and height increased with increasing age, especially between 8 and 13 years, and flexibility remained stable throughout childhood and adolescence.

Key words: Student; Flexibility; Maturation; Body composition. 


\section{INTRODUÇÃO}

Um importante componente da aptidão física relacionada à saúde é a flexibilidade, basicamente, ela é resultante da capacidade da elasticidade demonstrada pelos músculos e tecidos conectivos, combinados à mobilidade articular ${ }^{1}$.

Níveis adequados de flexibilidade têm sido associados à prevenção nos problemas posturais ${ }^{2}$, menor incidência de lesões ${ }^{3}$, principalmente, na região lombar e dorsal.

Fatores endógenos tais como o sexo, a idade, o somatótipo e a individualidade biológica, influenciam a flexibilidade. Esta, por sua vez, comporta-se de forma diferente em crianças $^{4,5}$, adolescentes ${ }^{6} \mathrm{e}^{\text {adultos }}{ }^{7,8} \mathrm{e}$ tende a diminuir com o aumento da idade ${ }^{5,8}$.

A flexibilidade, especificamente, até a puberdade, diminui e tende a aumentar, posteriormente, até atingir um platô e na idade adulta, tende diminuir ${ }^{9}, 10$. Difere, ainda, em função do sexo, sendo que as mulheres possuem maior flexibilidade comparada com os homens $s^{9-12}$.

Estudos das diferenças de flexibilidade entre os indivíduos têm levado em consideração fatores como medidas antropométricas ${ }^{13}$, composição corporal $^{11}$, genéticos ${ }^{4}$, culturais ${ }^{11}$ e patológicos ${ }^{9}$. Portanto, a caracterização da flexibilidade de um indivíduo é multifatorial e particularmente, fatores maturacionais e níveis de atividades físicas podem interagir na flexibilidade.

A indagação pelo entendimento dos índices de aptidão física relacionada à saúde em crianças e adolescentes tem tornado-se uma constante para permitir uma participação controlada e segura em programas de atividade física. Dessa forma, obtenção de informações relativas à flexibilidade em crianças e adolescentes, em particular no ambiente escolar, poderá contribuir para se ter valores de referência e providenciar comparações entre diversas regiões e estados brasileiros. Subsequentemente, poder-se-á acompanhar os efeitos dos exercícios de alongamento, juntamente com as alterações maturacionais.

Em face ao exposto, o presente estudo teve como objetivo avaliar e comparar a massa corporal, estatura, índice de massa corporal (IMC) e flexibilidade com a idade cronológica e a maturação sexual e, além disso, verificar a influência das variáveis citadas (idade cronológica, antropométricas, composição corporal e maturação sexual) na flexibilidade de escolares do sexo feminino da rede pública e particular de ensino do município de Cascavel, PR.

\section{PROCEDIMENTOS METODOLÓGICOS}

Trata-se de um estudo transversal realizado no ano de 2006 com escolares de oito a 17 anos de idade, do sexo feminino, da cidade de Cascavel/ $\mathrm{PR}$, regularmente matriculadas nas redes pública e particular de ensino.

A população de escolares deste estudo foi estimada em 39.830 mil. Para o calculo da amostra, foram utilizados os procedimentos propostos por Barbetta $^{14}$, admitindo um erro amostral de 1,89\%. Para a seleção da amostra, foi dividido o município em 3 pólos e selecionadas as turmas/alunos de forma aleatória simples, via sorteio por conglomerado.

Sendo assim, foi necessária a avaliação de 2663 escolares, destas, foram excluídas 59 da análise final: idade decimal inferior a oito e superior a 17 anos; as que se negaram a fazer alguma das avaliações; portadoras de doenças crônicas; deficientes físicos; com histórico médico de restrição ou uso de medicamentos que afetasse a aptidão física. Desta forma, a amostra final foi constituída de 2604 escolares, sendo $72,9 \%(n=1899)$ da rede pública e $27,1 \%(\mathrm{n}=705)$ da rede particular de ensino, divididos respectivamente: $3,2 \%$ e $4,4 \%$ ( 8 anos), 10,0\% e $11,5 \%$ ( 9 anos), $11,7 \%$ e $12,2 \%$ (10 anos), $14,2 \%$ e $12,1 \%$ ( 11 anos), $14,5 \%$ e $10,5 \%$ ( 12 anos), $12,9 \%$ e $11,2 \%$ (13 anos), $11,0 \%$ e $14,2 \%$ (14 anos), $9,8 \%$ e $12,3 \%$ ( 15 anos), $7,8 \%$ e $7,7 \%$ (16 anos) e $5,1 \%$ e 4,0\% (17 anos).

Todos os cinco profissionais de educação física envolvidos na avaliação física participaram de treinamento, para padronização das medidas, com o objetivo de obter maior precisão dos dados coletados. Cada profissional ficou responsável em medir apenas uma variável, durante os 40 dias de avaliação.

As variáveis analisadas neste estudo foram: Idade cronológica (I), massa corporal (MC), estatura (E), índice de massa corporal (IMC), flexibilidade (F) e maturação sexual (M).

As escolares foram pesadas em balança de Bio-Impedância Tanita ${ }^{\circledR}$ (modelo TBF 305), com graduação em 0,1 quilograma, usando roupas leves (camiseta e short) e descalças. A estatura foi mensurada com um estadiômetro de parede de marca Seca ${ }^{\circledR}$ com graduação em 1 mm ${ }^{15}$.

O Índice de Massa Corporal (IMC) foi obtido pela relação entre a massa corporal $(\mathrm{kg})$ dividido pela estatura $(\mathrm{m})$ elevada ao quadrado $(\mathrm{IMC}=$ massa corporal / estatura ${ }^{2}$ ).

A classificação das escolares quanto aos valores de IMC, realizou-se por meio da curva de distribui- 
ção, segundo percentil, baixo peso (> P5), eutróficos (P5 > P85), sobrepeso (P85 > P95) e obeso ( $\leq$ P95), pontos de corte propostos pelo National Center for Health Statistics - NCHS (CDC, 2000) ${ }^{16}$.

A flexibilidade foi mensurada utilizando-se o banco de "Wells", uma caixa de madeira com dimensões de 30,5 x 30,5 centímetros, tendo a parte superior plana com 56,5 centímetros de comprimento, na qual é fixada uma fita métrica, sendo que o valor $23 \mathrm{~cm}$ coincide com a linha onde o avaliado acomodava os pés. Este teste consiste em verificar a flexibilidade de tronco e dos músculos isquiotibiais. As escolares permaneciam sentadas com as pernas estendidas e os pés descalços, totalmente apoiados na caixa, com uma mão sobre a outra, mantendo os dedos unidos, indicadores sobrepostos e alinhados e apoiados sobre a superfície plana da caixa. Em seguida, com o joelho estendido, o escolar flexionava a coluna vertebral com a cabeça entre os braços até o alcance máximo do movimento, permanecer estático por aproximadamente dois segundos, enquanto o avaliador realizava a leitura na escala. As medidas foram realizadas três vezes, adotando o maior valor alcançado ${ }^{17}$.

Os pontos de corte adotados para a flexibilidade foram os propostos pelo projeto Esporte Brasil (PROESP-BR $)^{18}$, que classifica os resultados em três categorias: abaixo $(<23 \mathrm{~cm})$, dentro $(23-28 \mathrm{~cm})$ e acima $(>28 \mathrm{~cm})$ da zona de saúde e aptidão física.

Para a maturação sexual, utilizaram-se os critérios de estágios puberais propostos por Tanner ${ }^{19}$ para mamas (M1 a M5), sendo realizada autoavaliação, comparando as fotografias ilustrativas. Para este procedimento, as escolares foram orientadas individualmente, em uma sala previamente determinada, onde uma professora de educação física realizou a explanação da importância e dos objetivos da avaliação da maturação sexual e, em seguida, explicações sobre os procedimentos de auto-avaliação e o preenchimento do formulário. $\mathrm{Na}$ seqüência, foi distribuída a prancha com as fotografias dos cinco estágios de desenvolvimento das mamas, sendo solicitado que as escolares observassem com atenção cada uma das fotografias e marcassem no formulário de avaliação a que mais se parecia com elas naquele momento.

Primeiramente, foi realizada uma análise descritiva (frequência absoluta (n), relativa (\%), valores de média (M) e desvio-padrão (DP)) das variáveis de massa corporal, estatura, IMC e flexibilidade. A análise de variância (Anova Two-way) foi utilizada para comparar os grupos (idades e maturação sexual) em relação à massa corporal, estatura, IMC e flexibilidade, e para identificar as diferenças entre grupos, utilizou-se o teste de Post Hoc de Tukey. O coeficiente de correlação simples de Pearson foi utilizado para verificar a correlação da idade, massa corporal, estatura e IMC em relação à flexibilidade, para maturação sexual, foi utilizada a correlação de Spearman. Análise de regressão linear múltipla "stepwise" foi utilizada para determinar os possíveis efeitos de cada variável independente (idade, massa corporal, estatura, IMC e maturação sexual) em relação à dependente (flexibilidade). Para as variáveis qualitativas de idade e maturação sexual serem introduzidas no modelo de regressão múltipla, utilizaram-se as variáveis chamadas indicadoras. Todos os dados foram armazenados e analisados, utilizando-se o programa SPSS ${ }^{\circledR}$ for Windows (Statistical Package of Social Sciences) versão 13.0. Adotou-se nível de significância de $\mathrm{p}<0,05$ para todas as análises.

Este trabalho foi aprovado pelo Comitê de Ética em Pesquisa da Universidade Federal de Santa Catarina (UFSC), parecer $n^{\circ} 131 / 06$, tendo sido necessário o consentimento por escrito dos secretários de educação e dos diretores das escolas, autorizando a pesquisa no município e em cada escola assinatura dos pais ou responsáveis das escolares.

\section{RESULTADOS}

Em relação à variável de flexibilidade, dos 2604 escolares do sexo feminino avaliados, $34,8 \%$ estavam abaixo da zona de aptidão física, ou seja, não encostaram as mãos nas pontas dos pés, $29,8 \%$ estavam dentro da zona de aptidão física e 36,3\% encontravam-se acima da zona de aptidão física, desta forma, $65,2 \%$ alcançaram ou ultrapassaram as pontas dos pés.

Na Tabela 1, são apresentados os valores de média e desvio padrão das variáveis de massa corporal, estatura, IMC e flexibilidade. Não foram observadas diferenças estatisticamente significativas com o avanço da idade para a variável flexibilidade, já para massa corporal, estatura e IMC as diferenças estatisticamente significativas ocorreram nas idades de 8 a 13 anos quando comparados a um ano mais velho, observando, assim, um aumento gradual nos valores médios de massa corporal, estatura e IMC até os 13 anos de idade.

Verifica-se, na Tabela 2, um aumento gradual nos valores de massa corporal, estatura e IMC com o avanço dos estágios maturacionais, variações não observadas para variável de flexibilidade. Para massa corporal, estatura e IMC foram observadas 
Tabela 1. Média (M) e desvio padrão (DP) por idade das variáveis de massa corporal, estatura, IMC e flexibilidade.

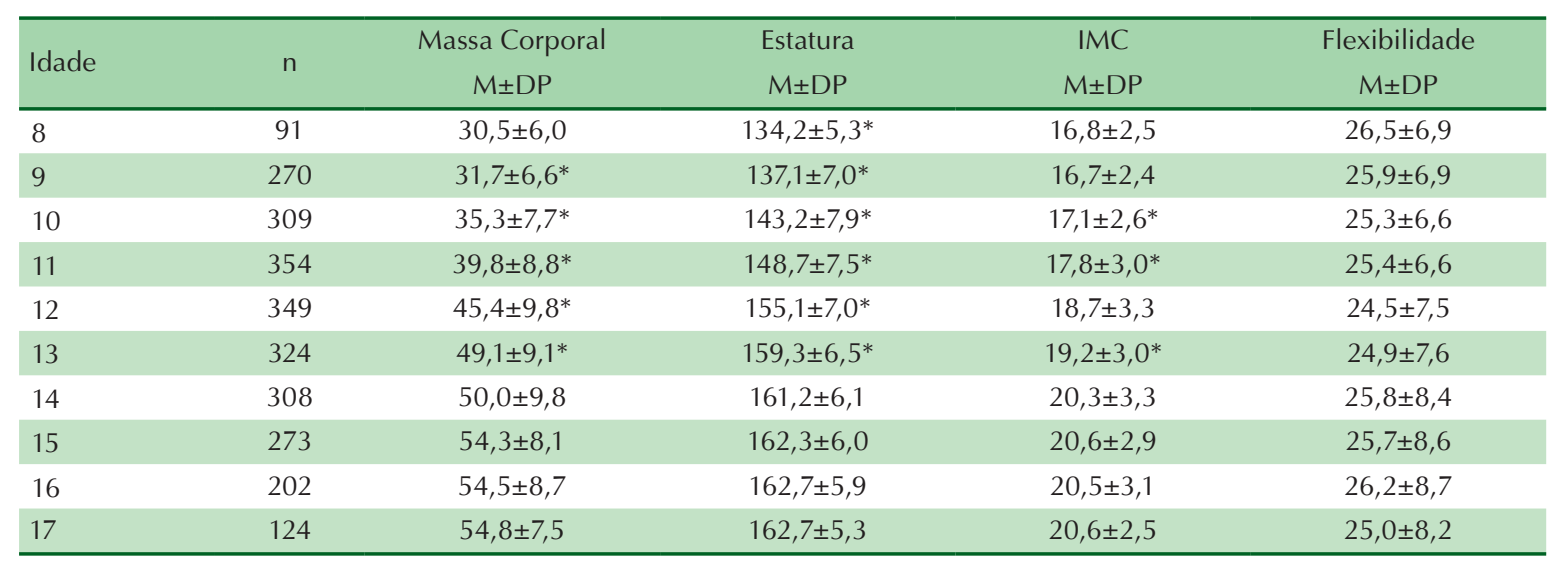

$* \mathrm{p}<0,05 \neq$ comparado a um ano mais velho.

Tabela 2. Média (M) e desvio padrão (DP) das variáveis de massa corporal, estatura, IMC e flexibilidade de acordo com os estágios maturacionais.

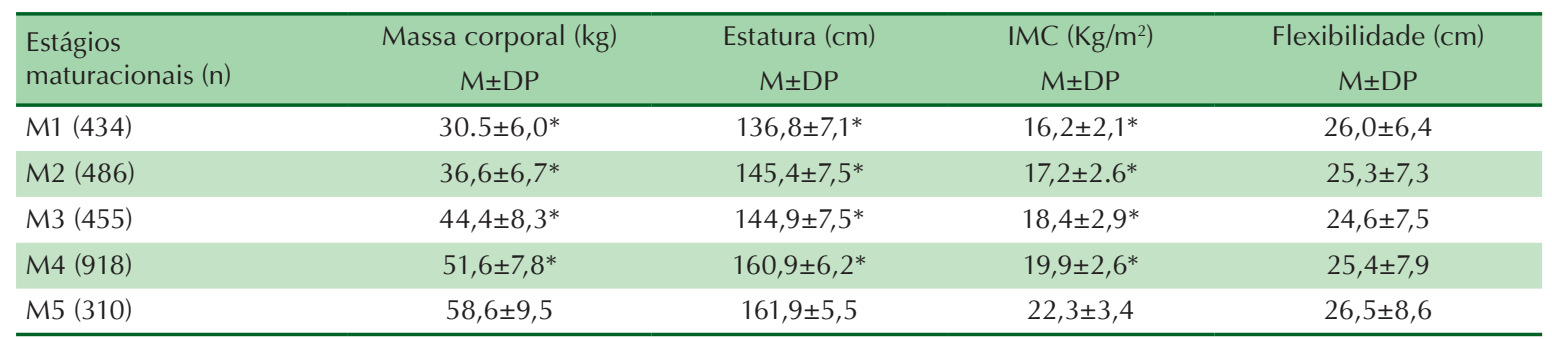

${ }^{*} \mathrm{p} \leq 0,05 \neq$ com o avanço dos estágios maturacionais.

diferenças inferiores e estatisticamente significativas em todos os estágios maturacionais quando comparados aos estágios mais avançados.

A análise de correlação linear de Pearson e Spearman é representada na Tabela 3. Observaramse correlações muito fracas $(r<0,16)$, para todas as variáveis independentes e estatisticamente significativas para as variáveis de estatura e IMC em relação à variável dependente (flexibilidade).

Tabela 3. Coeficiente de correlação da flexibilidade em relação às variáveis independentes de idade, massa corporal, estatura, IMC e maturação sexual.

\begin{tabular}{lccc}
\hline \multirow{2}{*}{ Variáveis independentes } & \multicolumn{3}{c}{ Flexibilidade } \\
& & $\mathrm{r}$ & $\mathrm{p}$ \\
\hline Idade (anos) & -0.02 & 0,91 \\
Massa corporal $(\mathrm{Kg})$ & Pearson & 0,15 & 0,45 \\
\hline Estatura $(\mathrm{m})$ & & $-0,06$ & 0,01 \\
\hline IMC $\left(\mathrm{kg} / \mathrm{m}^{2}\right)$ & & 0,06 & 0,00 \\
\hline Maturação sexual (estágios) & Spearman & 0,10 & 0,60 \\
\hline
\end{tabular}

Na Tabela 4, são apresentados os resultados da análise de regressão múltipla, nos quais se verifica que dentre as seis variáveis independentes inseridas na análise, somente três variáveis independentes (IMC, estatura e maturação sexual) apresentaram interação para predizer flexibilidade $\left(R^{2}=0,018\right)$. Esses resultados demonstraram que as variáveis independentes explicariam menos de $2 \%$ da variabilidade da flexibilidade.

\section{DISCUSSÃO}

De acordo com os resultados, observa-se que a massa corporal, estatura e IMC sofreram aumento crescente com o avanço da idade e estágios maturacionais. Já a flexibilidade permaneceu estável durante todos os períodos, tanto comparada à idade quanto a maturação sexual.

A flexibilidade é considerada um componente importante da aptidão física relacionada à saúde, destacada por estudiosos do assunto ${ }^{20}$, sendo parte importante da avaliação física, com diferentes métodos usualmente utilizados para avaliá-la ${ }^{8,20}$. De modo geral, o teste de sentar e alcançar, frequentemente utilizado em estudos epidemiológicos ${ }^{8}$, permite identificar a flexibilidade dos músculos da cadeia muscular posterior e a perda funcional dos mesmos e, ainda, acompanhar a evolução do indivíduo como indicador da função vertebral ${ }^{20}$. Sua acurácia foi comprovada por Baldaci et al. ${ }^{20}$, quando compararam três diferentes testes para 
Tabela 4. Resultados do modelo de regressão linear múltipla para a flexibilidade, após inclusão de seis variáveis independentes.

\begin{tabular}{|c|c|c|c|c|c|}
\hline \multirow{2}{*}{$\begin{array}{l}\text { Modelo de ordem } \\
\text { de entrada das variáveis }\end{array}$} & \multirow{2}{*}{ Estimativa } & \multicolumn{2}{|c|}{ Coeficiente } & \multicolumn{2}{|c|}{ IC $(95 \%)$} \\
\hline & & Erro Padrão & p-valor & $\mathrm{LI}$ & LS \\
\hline Constante & 22,452 & 0,863 & $p<0,000$ & 20,760 & 22,143 \\
\hline IMC & 0,162 & 0,045 & $p<0,000$ & 0,073 & 0,479 \\
\hline Constante & 31,494 & 1,988 & $p<0,000$ & 27,613 & 36,390 \\
\hline IMC & 0,264 & 0,049 & $p<0,000$ & 0,167 & 0,360 \\
\hline Estatura & $-0,072$ & 0,014 & $p<0,000$ & $-0,099$ & $-0,044$ \\
\hline \multicolumn{6}{|l|}{$3 R^{2}$ ajustado $=0,018$} \\
\hline Maturação & 0,569 & 0,202 & $\mathrm{p}<0,005$ & 0,173 & 0,964 \\
\hline
\end{tabular}

$R^{2}=$ Coeficiente de Variação; IC= Intervalo de Confiança; LS= Limite Superior; LI=Limite Inferior.

medir a flexibilidade dos músculos isquiotibiais de mulheres universitárias.

O método de avaliação da flexibilidade por meio do banco de Wells é comumente utilizado por professores de educação física nas escolas, buscando identificar características mecânicas e funcionais da flexibilidade anterior de tronco ${ }^{21}$. Sendo assim, torna-se importante a identificação dos índices em escolares, no sentido de melhor compreender os fatores intervenientes na flexibilidade, principalmente, durante o estirão de crescimento ${ }^{22}$.

Atualmente, verifica-se no Brasil um elevado número de estudos, caracterizando as condições de saúde dos escolares, no entanto, curvas regionais de normalidade relacionadas a indicadores de saúde, tais como composição corporal e flexibilidade, podem contribuir e ampliar os conhecimentos destas variáveis que estão fortemente atreladas ao ambiente, melhorando, assim, o diagnóstico e o acompanhamento do status de saúde dessa população.

Diferenças de flexibilidade entre os indivíduos têm levado em consideração fatores como medidas antropométricas ${ }^{6}$, composição corporal ${ }^{11}$, genéticos ${ }^{4}$, culturais ${ }^{11}$ e patológicos ${ }^{8}$, sendo assim a flexibilidade de um indivíduo é multifatorial ${ }^{5}$.

Segundo Lamari et al. ${ }^{5}$, maior comprimento dos membros superiores e inferiores indicam menores índices de flexibilidade em ambos os sexos. O mesmo ocorre com a estatura no sexo feminino, bem como na envergadura. Neste estudo, a massa corporal e a estatura não se correlacionam com o teste de sentar e alcançar. Esses achados são confirmados por Kravitz $z^{23}$ o qual observou que o comprimento dos membros superiores e inferiores, a envergadura, a massa corporal e a estatura não influenciaram significativamente na amplitude dos movimentos.

A composição corporal é outro fator que pode influenciar na flexibilidade. A concentração de tecido adiposo em torno das articulações pode vir a comprometer negativamente nos índices de flexibilidade ${ }^{21}$ No entanto, Bim $^{24}$ avaliou 29 meninas com idade de 16 e 17 anos, encontrando correlação significativa do IMC com a flexibilidade $(\mathrm{r}=$ 0,40 ), indicando que quanto maior o IMC maior a flexibilidade. $\mathrm{O}$ autor apresenta com hipótese esclarecedora para a correlação, aparentemente adversa, a pequena amplitude nos valores de IMC das adolescentes, e/ou ainda pelo fato de 93\% delas terem apresentado IMC desejáveis para a saúde, ou seja, entre os resultados obtidos, mesmo os mais altos valores encontram-se na faixa adequada à saúde.

Índices superiores de flexibilidade foram encontrados por Deforche et al. ${ }^{25}$ em meninas de 14 a 15 anos de idade, não obesas, em relação às obesas, levando em consideração que meninas com valores de percentual de gordura elevado tendem a antecipar a maturação sexual. Estas informações podem explicar, parcialmente, a diminuição da flexibilidade com o aumento da idade, justificando os achados de diversos estudos ${ }^{23,24}$.

Guedes e Guedes ${ }^{11}$ especificam que com o avanço da idade, principalmente, durante a puberdade, há uma relação inversa entre a espessura do tecido muscular e ósseo e a de gordura, ou seja, um acentuado ganho de massa muscular e óssea, com concomitante diminuição no ganho da massa de gordura. Autores ${ }^{5}$ justificam que as alterações na 
composição corporal que ocorrem com o avanço da idade, são relacionadas, principalmente, com alterações na musculatura, um dos componentes principais da capacidade motora geral, apontada pelos mesmos, como suposição elucidativa da diminuição da flexibilidade com o aumento da idade.

Corroborando os achados antropométricos e de composição corporal, o presente estudo verificou baixa correlação das variáveis, massa corporal $(\mathrm{r}=0,15)$, estatura $(\mathrm{r}=-0,06)$ e IMC $(\mathrm{r}=0,06)$ na flexibilidade.

Quando correlacionadas as variáveis de idade cronológica e maturação sexual com a flexibilidade, observaram-se correlações muito fracas $(r<0,10)$. Glaner ${ }^{26}$ considera ser a flexibilidade a variável que menos sofre variação dos 11 aos 17 anos de idade. Nesse sentido, Araújo e Oliveira ${ }^{27}$ não observaram diferenças na flexibilidade para ambos os sexos com o avanço da idade (10 a 14 anos), sugerindo, assim, que a idade não se correlaciona com a flexibilidade na infância e adolescência.

Dentre as variáveis de idade cronológica, massa corporal, estatura, IMC e maturação sexual, as que se correlacionaram estatisticamente significativas foram estatura e IMC. Deste modo, a equação sugerida na análise de regressão linear múltipla (flexibilidade $=37,423+(0,203 * I M C)+(-$ $0,114 *$ estatura $)+(0,569 *$ maturação $))$ não deve ser utilizada, devido a baixa predição das variáveis inseridas no modelo $\left(\mathrm{R}^{2}=0,018\right)$. Esses resultados não objetivaram substituir o teste de flexibilidade, mas apenas indicar as variáveis que, isoladas e/ou associadas, possam revelar variações na flexibilidade durante o período de crescimento, no sentido de contribuir na compreensão da flexibilidade.

A evolução do desempenho motor e físico na infância e adolescência está fortemente associada aos processos de crescimento e maturação ${ }^{10}$, principalmente, aqueles ocorridos durante o estirão de crescimento, momento no qual o indivíduo jovem tem um rápido aumento nas estruturas corporais. Sendo assim, as variações entre adolescentes de mesma idade cronológica são evidenciadas pelas diferenças na velocidade do processo de maturação biológica, no qual os jovens com desenvolvimento precoce apresentam um desempenho superior aos demais ${ }^{22}$.

A idade é destacada em alguns estudos como um fator interveniente na flexibilidade, e mulheres tendem a ser mais flexíveis do que os homens desde os cinco anos de idade, com acentuado aumento após a puberdade. A partir dessa fase, a velocidade de ganho é desacelerada, passando então a declinar, porém com progressão da diferença entre o sexo ${ }^{1}$.
Esta diferença pode estar associada ao surto de crescimento púbere, no qual os ossos longos acrescentam um índice de crescimento longitudinal mais acentuado que os músculos e tendões, recorrente das alterações hormonais, provocando uma diminuição temporária dos índices de flexibilidade, até que seja atingido o crescimento dessas estruturas $^{22}$.

Ao contrário dos resultados apresentados por outros autores, citados anteriormente, a flexibilidade das escolares deste estudo não sofreu variações significativas com o avanço da idade cronológica e estágios maturacionais.

De acordo com os pontos de corte propostos pelo PROESP-BR ${ }^{18}, 34,8 \%$ das escolares do presente estudo encontram-se abaixo da zona de saúde para a flexibilidade. No entanto, quando observados os valores de média por idade e maturação sexual, verifica-se que em todas as idades e níveis maturacionais os valores estão entre 23 e $28 \mathrm{~cm}$, ou seja, dentro da normalidade. Estes achados foram também confirmados por, Ulbrich et at. ${ }^{22}$ onde os resultados da flexibilidade para todos os estágios maturacionais estavam dentro da classificação, no qual os valores considerados ideais para indivíduos do sexo feminino de sete aos 17 anos devem ser entre 23 a $28 \mathrm{~cm}$.

Vários são os estudos avaliando a massa cor-

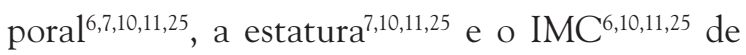
crianças $^{3-5,11,22,27,30}$ e adolescentes ${ }^{6,7,10,11,17,22,27}$ em relação à idade e maturação sexual, sendo observado aumento dos índices de flexibilidade com o avanço da idade e nível maturacional nestas variáveis. Corroborando estes resultados, a massa, a estatura e o IMC das escolares do presente estudo aumentaram com o avanço da idade e maturação sexual, principalmente, durante a puberdade.

Justifica-se este estudo em virtude da necessidade de se obter indicadores referenciais regionais confiáveis sobre a flexibilidade de escolares, podendo, assim, contribuir no trabalho dos vários profissionais da saúde tanto para prática clínica, como para investigações científicas e na elaboração das aulas de educação física.

$\mathrm{O}$ estudo indica que a etnia ${ }^{28}$ não influencia na flexibilidade. Porém, fatores não controlados no presente estudo, tais como o nível de habilidade motora, velocidade de execução e motivação do avaliado, na realização do teste, podem ter influenciado nesses índices, visto que atividades que envolvam alongamento podem aumentar ou manter os níveis de flexibilidade ${ }^{29}$, apesar destas limitações, isto não invalida os resultados apresentados. 
Estudos ${ }^{3,5,7,17}$ demonstram a necessidade de se produzirem novos conhecimentos com a finalidade de melhor compreender a boa saúde física de crianças e adolescentes.

Com isto, sugerem-se estudos longitudinais que avaliem as condições de saúde de escolares, controlando as limitações apresentadas, a fim de promover melhor entendimento acerca do crescimento e desenvolvimento desta população.

\section{CONCLUSÃO}

Com o avanço da idade e da maturação sexual, as variáveis de massa corporal, estatura e IMC sofrem um aumento crescente, principalmente, nas idades compreendidas entre oito e 13 anos.

Dentre os critérios de classificação da flexibilidade, mais de $65 \%$ das escolares atendem os critérios de normalidade estabelecidos pelo PROESP-BP.

A flexibilidade não sofreu influência das variáveis de idade, massa corporal, estatura, composição corporal e maturação sexual, mantendo-se estável por toda a infância e adolescência.

\section{REFERÊNCIAS BIBLIOGRÁFICAS}

1. Araújo CGS. Avaliação da Flexibilidade: Valores Normativos do Flexiteste dos 5 aos 91 Anos de Idade. Arq Bras Cardiol 2008;90(4):280-287.

2. Polachini LO, Fuzasaki L, Tamaso M, Tellini GG, Masieiro D. Estudo comparativo entre três métodos de avaliação do encurtamento de musculatura posterior da coxa. Rev Bras Fisiot 2005;9(2):187-193.

3. Rodríguez PL, Santonja FM, Lópesz-Mi PA, Sáinz de Baranda P, Yuste JL. Effect of physical education stretching programme on sit-and-reach score in schoolchildren. Sci Sports 2008;23(3):170-175

4. Grahame R. Time to take hypermobility seriously (in adults and children). Rheumatology 2001;40(5):485487.

5. Lamari NM, Chueire AG, Cordeiro JA. Analysis of joint mobility patterns among preschool children. São Paulo Med J 2005;123(3):119-123.

6. Conte M, Gonçalves A, Aragon FF, Padovani CR. Influência da massa corporal sobre a aptidão física em adolescentes: estudo a partir de escolares do ensino fundamental e médio de Sorocaba/SP. Rev Bras Med Sport 2000;6(2):44-49.

7. Feldman D, Shrier I, Rossignol M, Abenhaim L. Adolescent growth is not associated with changes in flexibility. Clin J Sport Med 1999;9:24-29.

8. Tsang YL, Mak MK. Sit-and-reach test can predict mobility of patients recovering from acute stroke. Arch Phys 2004;85(1):94-98.
9. Alaranta H, Hurri H, Heliovaara M, Soukka A, Harju R. Flexibility of the spine: normative values of goniometric and tape measurements. Scand J Rehabil Med 1994;26(3):147-154.

10. Re AHN. Relação entre crescimento, desempenho motor, maturação biológica e idade cronológica em jovens do sexo masculino. Rev Bras Educ Fis Esp 2005:19(2);153-162.

11. Guedes DP, Guedes JERP. Crescimento, composição o corporal e desempenho motor de crianças e adolescentes. São Paulo: CLR Baliero 1997.

12. Seckin U,Tur BS, Yilmaz O, Bodur H, Arasil T. The prevalence of hypermobility among high school student. Rheumatol Int 2005;25(4):260-263.

13. Grant S, Hasler T, Davies C, Aitchison TC, Wilson, Wittaker A. A comparison of the anthropometric, strength, endurance and flexibility characteristics of female elite and recreational climbers and non-climbers. J Sports Sci 2001;19(7):499-505.

14. Barbetta PA. Estatística aplicada às ciências sociais. $5^{\mathrm{a}}$ ed. Florianópolis: Ed. da UFSC, 2003. p.42-65.

15. Alvarez BR, Pavan AL. Alturas e Comprimentos. In: Petroski EL. editor. Antropometria: técnicas e padronizações. $2^{\mathrm{a}}$ ed. Porto Alegre: Palotti, 2003. p.31-44.

16. NCHS - National Center For Health Statistics. Centers for Disease Control and Prevention. Atlanta, GA: Department of Health and Human Services. Growth Charts: United States, 2000.

17. Mikkelsson LO, Nupponen H, Kaprio J, Kautiainen H, Mikkelsson M, Kujala UM. Adolescent flexibility, endurance strength, and physical activity as predictors of adult tension neck, low back pain, and knee injury: a 25 year follow up study. Br J Sports Med 2006;40(2):107-130.

18. Gaya ACA. PROESP-BR. Observatório Permanente dos Indicadores de saúde e fatores de prestação esportiva em crianças e jovens. Manual de aplicação de medidas e testes, normas e critérios de avaliação. Manual de Aplicação de Medidas e Testes Somatomotores, 2004;13.

19. Marshall WA, Tanner JM. Variation in the pattern of pubertal changes in girls. Arch Dis Child 1969;44:291303.

20. Baltaci G, Un N, Tunay V, Besler A, Gerceker S. Comparison of three different sit and reach tests for measurement of hamstring flexibility in female university students. Br J Sports Med 2003;37(1):59-61.

21. Silva DJL, Santos JAR, Oliveira BMPM. A flexibilidade em adolescentes - Um contributo para a avaliação global. Rev Bras Cineantropom Desempenho Hum 2006;8(1):72-79.

22. Ulbrich AZ, Bozza R, Machado HS, Michelin A, Vasconcelos IQA, Stabeline Neto A. Aptidão Física em crianças e adolescentes de diferentes estágios maturacionais. Fit Perf J 2007;6(5);277-282.

23. Kravitz L, Heyward VH. Flexibility Training - For the Full Health Benefits of Exercise Training, Don't Neglect Flexibility. Fitness Manage Mag 1995;11(2):32-38. 
24. Bim RH, Nardo Jr N. Aptidão física relacionada à saúde de adolescentes estagiários da Universidade Estadual de Maringá. Acta Sci Health Sci 2005; 27(1):77-85.

25. Deforche B, Lefevre J, De Bourdeaudhuij I, Hills AP, Duquet W, Bouckaert J. Physical fitness and physical activity in obese and nonobese flemish youth. Obes Res 2003;11(3):434-441.

26. Glaner MF. Aptidão Física relacionada à saúde de adolescentes rurais e urbanos em relação à critérios de referência. Rev Bras Educ Fis Esp 2005;20(1):13-24.

27. Araújo SS, Oliveira ACC. Aptidão Física de escolares de Aracajú. Rev Bras Cineantropom Desempenho Hum 2008;10(3):271-276.

28. Okano AH, Altimari LR, Dodero SR, Coelho AF, Almeida PBLA, Cyrino ES. Comparação entre o desempenho motor de crianças de diferentes sexos e grupos étnicos. Rev Bras Ciên Mov 2001;9(3):39-44.
29. Bagrichevsky M. O desenvolvimento da flexibilidade: uma análise teórica de mecanismos neurais intervenientes. Rev Bras Cienc Esp 2002;24(1):199-210.

\section{Endereço para correspondência}

Roberto Régis Ribeiro

Rua Pernambuco, 600

Edifício José Smarczewski, Ap. 801.

85810020 - Centro. Cascavel, PR. Brasil

E-mail: gisaminatto@hotmail.com/robertor@ fcm.unicamp.br 